\title{
Application of Möbius coordinate transformation in evaluating Newton's integral
}

\author{
Robert TENZER ${ }^{1}$, Vladislav GLADKIKH ${ }^{1}$ \\ ${ }^{1}$ National School of Surveying, Division of Sciences, University of Otago \\ 310 Castle Street, Dunedin, New Zealand; e-mail: Robert.Tenzer@otago.ac.nz
}

\begin{abstract}
We propose a numerical scheme which efficiently combines various existing methods of solving the Newton's volume integral. It utilises the analytical solution of Newton's integral for tesseroid in computing the near-zone contribution to gravitational field quantities (potential and its first radial derivative). The far-zone gravitational contribution is computed using the expressions derived based on applying Molodensky's truncation coefficients to a spectral representation of Newton's integral. The weak singularity of Newton's integral is treated analytically using formulas for the gravitational contribution of the cylindrical mass volume centered with respect to the observation point. All three solutions are defined and evaluated in the system of polar spherical coordinates. A conversion of the geographical to polar spherical coordinates of input data sets (digital terrain and density models) is based on the Möbius transformation with an enhanced integration grid resolution at vicinity of the observation point.
\end{abstract}

Key words: analytical integration, forward modelling, gravity field, Möbius transformation, Newton integral

\section{Introduction}

Various forward modelling techniques for computing the gravitational field quantities of a given density/mass distribution have been developed in the literature. These techniques utilise the numerical, semi-analytical, or analytical integration approaches. Anderson (1976) and others used a spherical prism (tesseroid) for an evaluation of the potential and its radial derivative. Martinec et al. (1993, 1994) and Martinec (1998) applied the semianalytical integration approach, where the analytical solution for the radial component of the integration domain was found from Bronstein and Semendjajew (1977), and an effective numerical integration scheme was employed for the surface integration in the system of spherical coordinates. 
Mikuška et al. (2006) applied a similar semi-analytical approach in the system of polar spherical coordinates. The analytical solutions for the potential and attraction of a spherical cap can be found in Kühtreiber et al. (1989). Tenzer et al. (2007) derived the analytical expressions for the potential and its first derivatives of the tesseroid of a homogeneous density defined in the system of polar spherical coordinates. Studying the local gravity field, a simple form of the integration element can be used, such as the right rectangular parallelepiped (prism) with a constant density within each individual integration volume. Bessel (1813) derived the closed analytical expression for the potential of a prism. The potential-related formulae for a prism were studied also by Zach (1811), Mollweide (1813), Everest (1830), and Mader (1951). Nagy et al. (2000) reviewed the closed analytical expressions for the potential and its first and second derivatives of the rectangular prism of a homogenous density.

For the gravitational field modelling of inhomogeneous density formations, the approximation of geological structures by more general geometrical forms are often implemented. Pick (1984) summarized the expressions for computing the gravitation of various geometrical bodies. Hurbbert (1948) introduced a methodology called the line-integral approach. This approach facilitates the expressions derived based on converting the surface or volume integrals into line integrals after applying the Gauss divergence theorem. Following this principle, Talwani et al. (1959) applied the line-integral approach to the polygon in 2-D. Talwani and Ewing (1960), Collette (1965), and Takin and Talwani (1966) decomposed the 3-D body into parallel, typically horizontal laminae. Paul (1974) and Barnett (1976) generalised this concept for a polyhedron in 3-D. Pohánka (1988) developed a simple algorithm for the gravitation of a homogeneous polyhedral body using the line-integral approach (see also Ivan, 1990; Pohánka, 1990). The expressions for polyhedral bodies with a homogeneous density were studied also by Okabe (1979), Götze and Lahmeyer (1988), Kwok (1991), Holstein and Ketteridge (1996), Werner and Scheeres (1997), Holstein et al. (1999), and Holstein (2002a,b). Petrovič (1996) presented in a more complete form the formulae for the potential and its derivatives using the line-integral approach for the arbitrary polyhedral bodies of a homogenous density (see also Tsoulis and Petrovič, 2001).

For the forward modelling of the gravitational field of geological struc- 
tures with a variable density distribution, the analytical expressions for volume elements with linearly or otherwise varying density distribution models can further improve the numerical efficiency. One example can be given by sedimentary basins where the density increases with depth due to compaction (e.g., Artemjev et al., 1994). Several authors derived and applied the analytical expressions of volume elements for various density distribution models. Chai and Hinze (1988) computed the gravitation using a rectangular prism with density changing linearly with depth. Gallardo-Delgado et al. (2003) derived the analytical solution for the gravitation utilising a right rectangular prism with density varying according to a polynomial quadratic law.

Garcia-Abdeslem (1992, 2005) introduced the analytical expression for the right rectangular prism with a depth-dependent density distribution having the form of a cubic polynomial. Karcol (2011) derived the expressions for computing the potential and its radial derivative of the spherical shell with a radially varying density. Combining benefits of using more generalized geometrical bodies and taking into account density variation models, Pohánka (1998) introduced the expression by means of the line integrals for computing the gravitation of an arbitrary polyhedral body having a linearly varying density. The alternative expression was derived by Hansen (1999). Holstein (2003) generalised their work deriving the formulae also for the potential and its second derivatives. To avoid singular terms and obtain a maximal numerical efficiency, Pohánka (1998) derived the optimum expression and proposed an efficient algorithm for computing the gravitation. Following his methodology, Hamayun et al. (2009) derived the corresponding optimum expression for the potential.

A number of authors have used the spectral representation of Newton's kernel in deriving the expressions for computing the gravitational effects of topography. Sünkel (1968) introduced the expressions for the potential in terms of the spherical height functions. Vanícek et al. (1995) formulated alternative expressions for the topographic potential and its radial derivative. Martinec et al. (1993), Martinec and Vaniček (1994), Sjöberg (1998), Sjöberg and Nahavandchi (1999), Sjöberg (2000), Tsoulis (1999), Novák (2000), Tsoulis (2001), Sjöberg (2001), Heck (2003), Tenzer (2005), Sjöberg (2007), Novák (2009), and others derived various spectral expressions for computing the topography-generated gravitational field quantities. 
Wild and Heck (2004) introduced the expressions for evaluating the topographic effect on satellite gradiometry data. Novák and Grafarend (2005) studied the topographic potential and its vertical gradient using the ellipsoidal representation of Newton's integral. Makhloof (2007) formulated the expressions for computing the topographic-isostatic effect on airborne gravimetry, satellite gravimetry, and gradiometry data. The alternative expressions for computing the topographic effects in satellite gravimetry and gradiometry were formulated by Novák and Grafarend (2006) and Eshagh and Sjöberg (2008, 2009). Studying the gravitational contribution of the far-zone topography, Novák et al. (2001) utilised various truncation coefficients to a spectral representation of Newton's integral. The alternative expressions for computing the far-zone contributions to gravity field quantities by means of Molodensky's truncation coefficients can be found in Tenzer et al. (2011).

In this study, we combine benefits of using the analytical integration approach for an accurate computation of the near-zone gravitational contribution, while the numerically efficient methods for a spherical harmonic analysis and synthesis of gravity field are employed in computing the gravitational contribution of the far-zone topography. Both, the analytical integration approach (for the near zone) and the spectral approach (for the far zone) are formulated uniformly in the system of polar spherical coordinates. The formulation of the problem is given in Section 2. A conversion of the geographical to polar spherical coordinates of the input data is based on the Möbius transformation. It takes into consideration a more refined integration grid in close proximity of the observation point. The coordinate transformation is described in Section 3. The analytical integration approach for computing the near-zone contribution utilises the expressions for the tesseroid mass volume. These analytical expressions are reviewed in Section 4. The expressions for computing the far-zone topography by means of spherical height functions are given in Section 5. The summary and concluding remarks are given in Section 6.

\section{Theory}

In spherical approximation, the topography-generated gravitational poten- 
tial $V$ computed at the position $(r, \Omega)$ is written in the following form

$$
\begin{aligned}
V(r, \Omega) & =\mathrm{G} \int_{0}^{2 \pi} \int_{0}^{\psi_{\text {inner }}} \rho(\alpha, \psi) \int_{\mathrm{R}}^{\mathrm{R}+H(\alpha, \psi)} \ell^{-1}\left(r, \psi, r^{\prime}\right) r^{\prime 2} \mathrm{~d} r^{\prime} \sin \psi \mathrm{d} \psi \mathrm{d} \alpha+ \\
& +\mathrm{G} \int_{0}^{2 \pi} \int_{\psi_{\text {inner }}}^{\psi_{0}} \rho(\alpha, \psi) \int_{\mathrm{R}}^{\mathrm{R}+H(\alpha, \psi)} \ell^{-1}\left(r, \psi, r^{\prime}\right) r^{\prime 2} \mathrm{~d} r^{\prime} \sin \psi \mathrm{d} \psi \mathrm{d} \alpha+ \\
& +\mathrm{G} \rho_{0} \int_{0}^{2 \pi} \int_{\psi_{0}}^{\pi} \int_{\mathrm{R}}^{\mathrm{R}+H(\alpha, \psi)} \ell^{-1}\left(r, \psi, r^{\prime}\right) r^{\prime 2} \mathrm{~d} r^{\prime} \sin \psi \mathrm{d} \psi \mathrm{d} \alpha
\end{aligned}
$$

where $\mathrm{G}=6.674 \times 10^{-11} \mathrm{~m}^{3} \mathrm{~kg}^{-1} \mathrm{~s}^{-2}$ is Newton's gravitational constant, $\mathrm{R}=6371 \times 10^{3} \mathrm{~m}$ is the Earth's mean radius (which approximates the geocentric radius of the geoid surface), $H$ is the topographical height, $\rho$ is the lateral topographic density function, $\rho_{0}$ is the mean topographical density, and $\ell$ is the Euclidean spatial distance between the observation point $(r, \Omega)$ and the integration (running) point $\left(r^{\prime}, \Omega^{\prime}\right)$. The 3 -D position is defined by geocentric spherical coordinates $(r, \phi, \lambda)$, where $r$ is the geocentric radius and $\Omega=(\phi, \lambda)$ denotes the geocentric direction with the geocentric spherical latitude $\phi$ and longitude $\lambda$. The surface integration domain in Eq. (1) is expressed in the system of polar spherical coordinates $(\alpha, \psi)$ with the spherical azimuth $\alpha$ and the spherical angle $\psi$. The relation between the spherical coordinates $(\phi, \lambda)$ and the polar spherical coordinates $(\alpha, \psi)$ is given by the well-known formulae of spherical trigonometry (e.g., Heiskanen and Moritz, 1967, Eqs. 2-207, 2-212)

$$
\begin{aligned}
& \cos \psi=\sin \phi \sin \phi^{\prime}+\cos \phi \cos \phi^{\prime} \cos \left(\lambda^{\prime}-\lambda\right), \\
& \tan \alpha=\frac{\cos \phi^{\prime} \sin \left(\lambda^{\prime}-\lambda\right)}{\cos \phi \sin \phi^{\prime}-\sin \phi \cos \phi^{\prime} \cos \left(\lambda^{\prime}-\lambda\right)} .
\end{aligned}
$$

The gravitational potential $V$ and acceleration $g$ are continuous at the entire 3-D space and they are regular at infinity (cf. Kellogg, 1929). Newton's integral is singular when the integration point coincides with the observation point (i.e., $\psi \rightarrow 0 \wedge r^{\prime} \rightarrow r$ ). Therefore, special care should be taken when calculating the contribution to the potential and acceleration from the 
integration area at vicinity of the observation point. However, the singularity of Newton's integral is weak and thus removable (cf. Kellogg, 1929, p. 151), see also Martinec (1998). The surface integration area in Eq. (1) is subdivided into three sub-domains. The first integral term on the righthand side of Eq. (1), bounded by the maximum spherical distance $\psi_{\text {inner }}$, represents the inner zone which contains the singularity. There are several possible methods of solving a weak singularity of Newton's integral. In our particular case, we propose the solution based on the analytical expressions for the cylinder mass volume centred at the observation point. Since the entire surface integration area in Eq. (1) is subdivided unambiguously into the inner zone (for solving the weak singularity), near zone, and far zone, this choice of the volumetric element for solving the singularity problem fits very well with the adopted integration scheme formulated in the system of polar spherical coordinates. The second integral on the right-hand side of Eq. (1) represents the gravitational contribution of the near-zone topography. The near-zone surface integration area is limited by the maximum spherical distance $\psi_{0}$. The gravitational contribution of the far-zone topography is given by the third integral in Eq. (1). Whereas the lateral density distribution $\rho$ is considered for the near zone, the constant mean value of the topographic density $\rho_{0}$ is assumed for computing the far-zone contribution. We further denote the gravitational potential generated by the near-zone topography as $V_{\mathrm{nz}}$ and the far-zone topographical potential as $V_{\mathrm{fz}}$.

The numerical scheme comprises the following steps: First, geographical coordinates of the elevation and density data (within the near zone) are converted to polar spherical coordinates using the Möbius transformation (see Section 3). The evaluation of the elevation and density values on the integration grid from the corresponding data grid (in the system of polar spherical coordinates) is done using, for instance, the bilinear interpolation. The surface integration zones, the analytical integration step within the near zone, and the maximum degree of spherical height functions for computing the far-zone contribution are chosen depending on the spatial data grid resolution and the required accuracy. We note that the choice of these parameters for an efficient integration requires additional numerical analysis not discussed in detail in this study. The computation of the gravitational field quantities is finally realised according to the expressions given in Sections 4 and 5 (and Appendix I). 


\section{Coordinate transformation}

The spherical coordinates $(\phi, \lambda)$ of input data grids are converted to the polar spherical coordinates $(\psi, \alpha)$ of which the origin is reckoned relative to the observation point. We note here that if input data are provided on a grid of geodetic coordinates, the additional conversion of geodetic latitude $\varphi$ to spherical latitude $\phi$ is required in prior of computing the polar spherical coordinates. The conversion formula between $\varphi$ and $\phi$ reads $\tan \varphi=\tan \phi(1-\mathrm{f})^{-2}$, where $\mathrm{f}$ is the linear flattening of the reference ellipsoid (e.g., Bomford, 1962). Bentsen et al. (1999) presented the transformation scheme, where the north and south poles are mapped onto arbitrary locations on the sphere. The transformation of spherical to polar spherical coordinates is performed in three successive steps, namely the stereographic projection from the unit sphere onto a complex plane, then a Möbius transformation of the complex plane, and finally a reverse stereographic projection from the complex plane onto the unit sphere. Both, the stereographic projection and the Möbius transformation are conformal. Therefore, the resulting three step transformation procedure is also conformal and thus preserves the angles at which curves cross each other. In particular, the orthogonal coordinate system on the sphere is transformed to a coordinate system which is also orthogonal. The direct transformation is given by the following scheme

$$
(\theta, \lambda) \stackrel{z=\tan \left(\frac{\theta}{2}\right) \mathrm{e}^{i \lambda}}{\longrightarrow} z \stackrel{w=\frac{(z-a)(c-b)}{(z-b)(c-a)}}{\longrightarrow} w \stackrel{\psi=2 \arctan |w|, \alpha=\arg (w)}{\longrightarrow}(\psi, \alpha) .
$$

The inverse transformation is described by

$$
(\psi, \alpha) \stackrel{w=\tan \left(\frac{\psi}{2}\right) \mathrm{e}^{\mathrm{i} \alpha}}{\longrightarrow} w \stackrel{z=\frac{-w b(c-a)+a(c-b)}{-w(c-a)+c-b}}{\longrightarrow} z \stackrel{\theta=2 \arctan |z|, \lambda=\arg (z)}{\longrightarrow}(\theta, \lambda) .
$$

In Eqs. (4) and (5), $a, b$, and $c$ are the complex constants; $z$ and $w$ are the complex variables; and $\theta=\pi / 2-\phi$ is the spherical co-latitude. The Möbius transformation is uniquely defined by mapping three arbitrary points. To achieve a more refined integration grid in close proximity of the observation point while increasing the numerical efficiency by decreasing the integration step with an increasing distance from the observation point, we specify the 


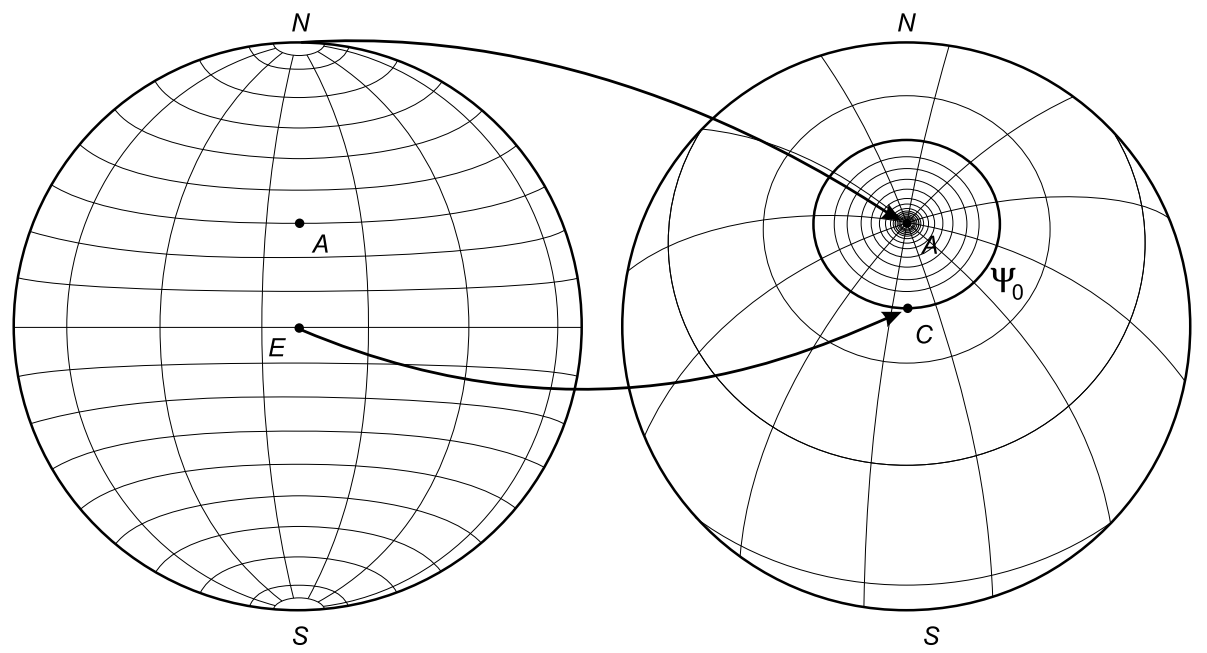

Fig. 1. The principle of the Möbius coordinate transformation defined in Eqs. (4-12). N - north pole, $\mathrm{S}$ - south pole, $\mathrm{A}$ - observation point, $\mathrm{E}$ - point in the intersection of the equator and the meridian of the observation point. During the transformation, the point $\mathrm{E}$ is mapped to $\mathrm{C}$. The (conventional) spherical distance between $\mathrm{A}$ and $\mathrm{C}$ is denoted as $\psi_{0}$.

Möbius transformation as follows: The north pole of the coordinate system is mapped onto the location of the observation point and the south pole is mapped onto the point diametrically opposite to the observation point. The third point on the Earth's surface is chosen exactly between the north and south poles on the meridian that passes through the observation point. The image of this point is on the same meridian but its latitude is shifted by the value of $\psi_{0}$ from the observation point. This means that during the transformation, the entire northern hemisphere is mapped onto the near zone enclosed by the spherical distance $\psi_{0}$ from the observation point. The situation is demonstrated in Fig. 1. We denote the spherical coordinates of the observation point as $\left(\theta_{a}, \lambda_{a}\right)$, the corresponding coordinates of the point on the opposite side of the Earth as $\left(\theta_{b}, \lambda_{b}\right)$, and the coordinates of the point between these two points as $\left(\theta_{c}, \lambda_{c}\right)$. We then write

$\theta_{b}=\pi-\theta_{a}, \quad \lambda_{b}=\pi+\lambda_{a}$.

Moreover, for the coordinates $\left(\theta_{c}, \lambda_{c}\right)$ we have 
$\theta_{c}= \begin{cases}\theta_{a}+\psi_{0}, & \theta_{a}+\psi_{0} \leq \pi \\ 2 \pi-\theta_{a}-\psi_{0}, & \theta_{a}+\psi_{0} \geq \pi\end{cases}$

and

$\lambda_{c}= \begin{cases}\lambda_{a}, & \theta_{a}+\psi_{0} \leq \pi \\ \pi+\lambda_{a}, & \theta_{a}+\psi_{0} \geq \pi\end{cases}$

The complex parameters $a, b$, and $c$ in Eqs. (4) and (5) are defined as

$a=\tan \left(\frac{\theta_{a}}{2}\right) e^{i \lambda_{a}}, \quad b=-\frac{1}{\bar{a}}, \quad c=\frac{a+d}{1-a \bar{d}}$,

where $\bar{a}$ and $\bar{d}$ are the complex conjugates of $a$ and $d$, respectively. The parameter $d$ reads

$d=e^{i \lambda_{a}} \tan \frac{\psi_{0}}{2}$.

The spherical distance $\psi$ and azimuth $\alpha$ are found by substituting the parameters $a, b$, and $c$ from Eq. (9) to Eqs. (4) and (5). We obtain

$$
\begin{aligned}
\cos \psi & =\cos (2 \arctan |w|)=\frac{1-|w|^{2}}{1+|w|^{2}}= \\
& =\frac{\cos \theta \cos \theta_{a}+\sin \theta \sin \theta_{a} \cos \left(\lambda-\lambda_{a}\right)-\cos \psi_{0}}{1-\cos \psi_{0}\left[\cos \theta \cos \theta_{a}+\sin \theta \sin \theta_{a} \cos \left(\lambda-\lambda_{a}\right)\right]},
\end{aligned}
$$

where

$w=\frac{z-a}{d(z \bar{a}+1)}$.

For $\psi_{0}=\pi / 2, \cos \psi_{0}=0$ and the expression in Eq. (11) becomes identical with the expression given in Eq. (2).

\section{Analytical integral approach for the near-zone contribution}

The near-zone contribution to the potential $V_{\mathrm{nz}}$ is computed as a sum of individual contributions of the tesseroid mass volumes $\left\{V_{j}^{\text {tes }}: j=1,2, \ldots, \mathrm{J}\right\}$. Hence 
$V_{\mathrm{nz}}=\sum_{j=1}^{\mathrm{J}} V_{j}^{\mathrm{tes}}$

where $\mathrm{J}$ is the total number of tesseroid elements within the near zone. The potential $V^{\text {tes }}$ generated by the individual tesseroid is given by

$V^{\mathrm{tes}}=\mathrm{G} \rho \int_{\alpha=\alpha_{1}}^{\alpha_{2}} \int_{\psi=\psi_{1}}^{\psi_{2}} \int_{r^{\prime}=r_{1}^{\prime}}^{r_{2}^{\prime}} \ell^{-1}\left(r, \psi, r^{\prime}\right) r^{\prime 2} \mathrm{~d} r^{\prime} \sin \psi \mathrm{d} \psi \mathrm{d} \alpha$.

The volume element is given by $\Delta v=r^{\prime 2} \Delta r^{\prime} \sin \psi \Delta \psi \Delta \alpha$, where $\Delta \psi=$ $\psi_{2}-\psi_{1}, \Delta \alpha=\alpha_{2}-\alpha_{1}$, and $\Delta r^{\prime}=r_{2}^{\prime}-r_{1}^{\prime}$. Furthermore, we assume a homogeneous mass density distribution $\rho$ within the volume of each tesseroid. The analytical solution of Newton's integral in Eq. (14) is given by (Tenzer et al., 2007)

$V^{\mathrm{tes}}=\left.\left.\left.\mathrm{G} \rho \mathrm{F}_{\alpha, \psi, r^{\prime}}\right|_{r_{1}^{\prime}} ^{r_{2}^{\prime}}\right|_{\psi_{1}} ^{\psi_{2}}\right|_{\alpha_{1}} ^{\alpha_{2}}$.

where the function $\mathrm{F}_{\alpha, \psi, r^{\prime}}$ reads

$$
\begin{aligned}
F_{\alpha, \psi, r^{\prime}} & =\alpha\left[\frac{1}{3 r} \ell^{3}\left(r, \psi, r^{\prime}\right)+\frac{1}{2} \ell\left(r, \psi, r^{\prime}\right) \cos \psi\left(r^{\prime}-r \cos \psi\right)+\right. \\
& \left.+\frac{r^{2}}{2} \cos \psi \sin ^{2} \psi \ln \left|2 \ell\left(r, \psi, r^{\prime}\right)+2 r^{\prime}-2 r \cos \psi\right|\right] \quad(r \neq 0) .
\end{aligned}
$$

By analogy with Eq. (15), the acceleration $g^{\text {tes }}$ generated by the tesseroid is defined as (Tenzer et al., 2007)

$$
g^{\mathrm{tes}} \cong-\frac{\partial V^{\mathrm{tes}}}{\partial r}=-\left.\left.\left.\mathrm{G} \rho \frac{\partial F_{\alpha, \psi, r^{\prime}}}{\partial r}\right|_{r_{1}^{\prime}} ^{r_{2}^{\prime}}\right|_{\psi_{1}} ^{\psi_{2}}\right|_{\alpha_{1}} ^{\alpha_{2}} \text {. }
$$

The radial derivative of $\mathrm{F}_{\alpha, \psi, r^{\prime}}$ in Eq. (17) reads

$$
\begin{aligned}
\frac{\partial F_{\alpha, \psi, r^{\prime}}}{\partial r} & =\alpha\left[r \cos \psi \sin ^{2} \psi \ln \left|2 \ell\left(r, \psi, r^{\prime}\right)+2 r^{\prime}-2 r \cos \psi\right|+\right. \\
& +\frac{\ell\left(r, \psi, r^{\prime}\right)}{6 r^{2}}\left(4 r^{2}-2 r^{\prime 2}-2 r r^{\prime} \cos \psi-3 r^{2} \cos ^{2} \psi\right)+
\end{aligned}
$$




$$
\begin{aligned}
& +\frac{\cos \psi}{2 \ell\left(r, \psi, r^{\prime}\right)}\left(r r^{\prime} \cos ^{2} \psi-r^{2} \cos \psi-r^{2} \cos \psi+r r^{\prime}\right)+ \\
& \left.+\frac{1}{2} \frac{r^{2} \cos \psi \sin ^{2} \psi}{\ell\left(r, \psi, r^{\prime}\right)}\left(\frac{r-\ell\left(r, \psi, r^{\prime}\right) \cos \psi-r^{\prime} \cos \psi}{\ell\left(r, \psi, r^{\prime}\right)+r^{\prime}-r \cos \psi}\right)\right] \\
& \quad\left(r \neq 0 ; r \neq r^{\prime} \wedge \psi \neq 0\right) .
\end{aligned}
$$

The parameters $\Delta \psi$ and $\Delta \alpha$ of the analytical integration step within the inner zone are chosen based on the required accuracy and the spatial resolution of the digital terrain and density models. We note here that the equivalent accuracy of input data and computed results (within the near zone) can be achieved if the input data and analytical integration have the same or similar spatial resolution. The maximum spherical distance $\psi_{\text {inner }}$ of the inner zone (for solving the singularity) can then be chosen as $\Delta \psi \approx \psi_{\text {inner }}$. The maximum spherical distance $\psi_{0}$ of the near zone can be selected based on the analysis of results computed for different values of $\psi$.

\section{Spectral approach for the far-zone contribution}

Novák (2010, Eq. 20) derived the expression for computing the potential $V$ in the following spectral form

$$
V(r, \Omega)=\frac{\mathrm{GM}}{\mathrm{R}} \sum_{n=0}^{\bar{n}} \sum_{m=-n}^{n}\left(\frac{\mathrm{R}}{r}\right)^{n+1} V_{\mathrm{n}, \mathrm{m}} Y_{\mathrm{n}, \mathrm{m}}(\Omega),
$$

where

$$
V_{\mathrm{n}, \mathrm{m}}=\frac{3}{2 n+1} \frac{\rho_{0}}{\bar{\rho}^{\mathrm{Earth}}} F_{\mathrm{n}, \mathrm{m}} .
$$

From Eq. (19), the expression for computing the acceleration $g$ is found to be

$$
\begin{aligned}
g(r, \Omega) & \cong-\frac{\partial V(r, \Omega)}{\partial r}= \\
& =\frac{\mathrm{GM}}{\mathrm{R}^{2}} \sum_{n=0}^{\bar{n}}(n+1) \sum_{m=-n}^{n}\left(\frac{\mathrm{R}}{r}\right)^{n+2} V_{\mathrm{n}, \mathrm{m}} Y_{\mathrm{n}, \mathrm{m}}(\Omega) .
\end{aligned}
$$


The average density of the upper continental crust $2670 \mathrm{~kg} / \mathrm{m}^{3}$ (cf. Hinze, 2003) is typically adopted as the mean topographical mass density $\rho_{0}$ in Eq. (20), and the value of $\bar{\rho}^{\text {Earth }}=5500 \mathrm{~kg} / \mathrm{m}^{3}$ is considered for the Earth's mean mass density. The geocentric gravitational constant is defined by the value $\mathrm{GM}=3986005 \times 10^{8} \mathrm{~m}^{3} / \mathrm{s}^{2}$. The maximum degree of spherical harmonics is denoted as $\bar{n}$. The surface spherical harmonic functions $\mathrm{Y}_{\mathrm{n}, \mathrm{m}}$ in Eqs. (19) and (21) read (e.g., Hobson, 1931)

$\mathrm{Y}_{\mathrm{n}, \mathrm{m}}(\Omega)=\mathrm{P}_{\mathrm{n}, \mathrm{m}}(\sin \phi)\left\{\begin{array}{ll}\cos m \lambda & (m \geq 0) \\ \sin |m| \lambda & (m<0)\end{array}\right.$,

where $\mathrm{P}_{\mathrm{n} . \mathrm{m}}$ are the Legendre associated functions of degree $n$ and order $m$ for the argument of sine of the spherical latitude $\phi$. The numerical coefficients $F_{\mathrm{n}, \mathrm{m}}$ in Eq. (20) are given by (Novák, 2010)

$$
\begin{aligned}
F_{\mathrm{n}, \mathrm{m}} & =\frac{\mathrm{H}_{\mathrm{n}, \mathrm{m}}}{\mathrm{R}}+(n+2) \frac{\mathrm{H}_{\mathrm{n}, \mathrm{m}}^{(2)}}{2 \mathrm{R}^{2}}+(n+2)(n+1) \frac{\mathrm{H}_{\mathrm{n}, \mathrm{m}}^{(3)}}{6 \mathrm{R}^{3}}+ \\
& +(n+2)(n+1) n \frac{\mathrm{H}_{\mathrm{n}, \mathrm{m}}^{(4)}}{24 \mathrm{R}^{4}}+(n+2)(n+1) n(n-1) \frac{\mathrm{H}_{\mathrm{n}, \mathrm{m}}^{(5)}}{120 \mathrm{R}^{5}}+ \\
& +\sum_{k=5}^{n+2}\left(\begin{array}{l}
n+2 \\
k
\end{array}\right) \frac{(-1)^{k}}{k+1} \frac{\mathrm{H}_{\mathrm{n}, \mathrm{m}}^{(k+1)}}{\mathrm{R}^{\mathrm{k}+1}},
\end{aligned}
$$

where $\mathrm{H}_{\mathrm{n}, \mathrm{m}}$ are the global elevation model (GEM) coefficients. The GEM coefficients $H_{\mathrm{n}, \mathrm{m}}$ describe the global topography. The series $\sum_{\mathrm{m}=-\mathrm{n}}^{\mathrm{n}} \mathrm{H}_{\mathrm{n}, \mathrm{m}} \mathrm{Y}_{\mathrm{n}, \mathrm{m}}$ defines the surface spherical height function $\mathrm{H}_{\mathrm{n}}$ of degree $n$ (e.g., Novák, 2000)

$$
\begin{aligned}
\mathrm{H}_{\mathrm{n}}(\Omega) & =\frac{2 n+1}{4 \pi} \int_{0}^{2 \pi} \int_{0}^{\pi} H(\psi, \alpha) P_{n}(\cos \psi) \sin \psi \mathrm{d} \psi \mathrm{d} \alpha= \\
& =\sum_{\mathrm{m}=-\mathrm{n}}^{\mathrm{n}} \mathrm{H}_{\mathrm{n}, \mathrm{m}} \mathrm{Y}_{\mathrm{n}, \mathrm{m}}(\Omega),
\end{aligned}
$$

where $\mathrm{P}_{\mathrm{n}}$ is the Legendre polynomial of degree $n$. Consequently,

$\mathrm{H}_{\mathrm{n}}^{(\mathrm{i})}(\Omega)=\sum_{\mathrm{m}=-\mathrm{n}}^{\mathrm{n}} \mathrm{H}_{\mathrm{n}, \mathrm{m}}^{(\mathrm{i})} \mathrm{Y}_{\mathrm{n}, \mathrm{m}}(\Omega)$. 
To derive the expressions for the gravitational field quantities generated by the far-zone topography of a constant mean density $\rho_{0}$, we define the farzone spherical height functions $\tilde{\mathrm{H}}_{\mathrm{n}}$ in the following form (cf. Tenzer et al., 2011)

$$
\begin{aligned}
\tilde{\mathrm{H}}_{\mathrm{n}}\left(\Omega, \psi_{0}\right) & =\frac{2 n+1}{4 \pi} \int_{0}^{2 \pi} \int_{\psi_{0}}^{\pi} H(\psi, \alpha) P_{n}(\cos \psi) \sin \psi \mathrm{d} \psi \mathrm{d} \alpha=(n-1) \times \\
& \times \frac{\mathrm{Q}_{\mathrm{n}}\left(\psi_{0}\right)}{2} \frac{2 n+1}{4 \pi} \int_{0}^{2 \pi} \int_{0}^{\pi} H(\psi, \alpha) P_{n}(\cos \psi) \sin \psi \mathrm{d} \psi \mathrm{d} \alpha .
\end{aligned}
$$

The Molodensky's truncation coefficients $\mathrm{Q}_{\mathrm{n}}$ for the argument $\psi_{0}$ read (Molodensky et al., 1960)

$\mathrm{Q}_{\mathrm{n}}\left(\psi_{0}\right)=\int_{\psi_{0}}^{\pi} S(\psi) \mathrm{P}_{\mathrm{n}}(\cos \psi) \sin \psi \mathrm{d} \psi$

where $S$ is the Stokes function. The coefficients $\mathrm{Q}_{\mathrm{n}}$ are computed recurrently according to formulae provided by Hagiwara (1975). Alternatively, they can be computed using the algorithm developed by Paul (1973). From Eqs. (24) and (26), the relation between the far-zone and original spherical height functions $\tilde{\mathrm{H}}_{\mathrm{n}}$ and $\mathrm{H}_{\mathrm{n}}$ is defined as

$$
\begin{aligned}
\tilde{\mathrm{H}}_{n}\left(\Omega, \psi_{0}\right) & =(n-1) \frac{\mathrm{Q}_{\mathrm{n}}\left(\psi_{0}\right)}{2} \mathrm{H}_{n}(\Omega)= \\
& =(n-1) \frac{\mathrm{Q}_{\mathrm{n}}\left(\psi_{0}\right)}{2} \sum_{\mathrm{m}=-\mathrm{n}}^{\mathrm{n}} \mathrm{H}_{\mathrm{n}, \mathrm{m}} \mathrm{Y}_{\mathrm{n}, \mathrm{m}}(\Omega) .
\end{aligned}
$$

As seen from Eq. (28), $\tilde{\mathrm{H}}_{\mathrm{n}}$ and $\mathrm{H}_{\mathrm{n}}$ are functionally related by means of Molodensky's truncation coefficients. The expression for computing the far-zone contribution to the potential $V_{\mathrm{fz}}$ (the third term on the right-hand side of Eq. 1) is obtained after applying the relation between $\tilde{\mathrm{H}}_{\mathrm{n}}$ and $\mathrm{H}_{\mathrm{n}}$ in Eq. (28) to the spectral representation of the potential $V$ in Eq. (19). We get

$$
V_{\mathrm{fz}}(r, \Omega)=\frac{\mathrm{GM}}{2 \mathrm{R}} \sum_{n=0}^{\bar{n}}(n-1) \mathrm{Q}_{\mathrm{n}}\left(\psi_{0}\right) \sum_{m=-n}^{n}\left(\frac{\mathrm{R}}{r}\right)^{n+1} V_{\mathrm{n}, \mathrm{m}} Y_{\mathrm{n}, \mathrm{m}}(\Omega) .
$$


From Eqs. (21) and (28), the expression for computing the acceleration generated by the far-zone topography $g_{\mathrm{fz}}$ is obtained in the following form

$$
\begin{aligned}
g_{\mathrm{fz}}(r, \Omega) & =\frac{\mathrm{GM}}{2 \mathrm{R}^{2}} \sum_{n=0}^{\bar{n}}(n-1)(n+1) \mathrm{Q}_{\mathrm{n}}\left(\psi_{0}\right) \times \\
& \times \sum_{m=-n}^{n}\left(\frac{\mathrm{R}}{r}\right)^{n+2} V_{\mathrm{n}, \mathrm{m}} Y_{\mathrm{n}, \mathrm{m}}(\Omega) .
\end{aligned}
$$

Equations (29) and (30) define the far-zone contributions to the topographic potential and acceleration by means of Molodensky's truncation coefficients. The far zone is specified by the parameter $\psi_{0}$ of Molodensky's truncation coefficients.

The currently available global elevation models such as the global topographic/bathymetric model DTM2006.0 (publicly released together with EGM2008 by the U.S. National Geospatial-Intelligence Agency EGM development team) allow computing the far-zone contribution to a very high accuracy and spectral resolution (up to degree 2160 of spherical harmonics). Since the accuracy of computed gravitational field quantities mainly depends on the accuracy of the input data and integration methods applied within the near zone, the computational time efficiency can be achieved by a proper selection of the maximum degree $\bar{n}$ of the GEM coefficients in computing the contribution of the far-zone topography.

\section{Summary and concluding remarks}

We have proposed and discussed the forward modelling technique for computing the topography-generated gravitational potential and its first radial derivative in the system of polar spherical coordinates. The integration scheme utilises three different integration approaches specified individually for the inner zone, near zone, and far zone.

The weak singularity of Newton's integral is solved analytically within the inner zone using the expressions for the cylinder mass volume centered with respect to the observation point. The choice of this particular geometric body is convenient for the analytical integration scheme in the system of polar spherical coordinates due to the fact that the upper limit (for inner 
zone) and lower limit (for near zone) of the surface integration domains are defined uniquely by the parameter $\psi_{\text {inner }}$. Similarly, the upper limit (for near zone) and lower limit (for far zone) of the surface integration sub-domains are uniquely defined by the parameter $\psi_{0}$ of the spherical distance. The near-zone integration is done analytically using the closed expressions for tesseroid. The analytical integration approach reduces the errors otherwise presented when using the numerical or semi-analytical integral approaches. The formulation of the analytical integration in the spherical approximation also reduces the errors due to the planar approximation presented when applying, for instance, the commonly used analytical rectangular prism approach. Moreover, possible errors caused by an ambiguous definition of the integration sub-domains in the case of using different integration schemes (for each sub-domain) are completely eliminated. The proposed conversion of geographical coordinates of input data sets (digital terrain and density models) to the integration grid of polar spherical coordinates, based on the Möbius transformation and subsequent data interpolation, has the enhanced resolution of the analytical integration grid at vicinity of the observation point while this resolution decreases with an increasing distance from the observation point. The gravitational contribution of the far-zone topography is computed using expressions which utilise the far-zone spherical height functions defined by means of Molodensky's truncation coefficients.

The size of the near zone (defined by the parameter $\psi_{0}$ ), the resolution of analytical integration within the inner zone (defined by the size of $\Delta \psi$ and $\Delta \alpha$ ), and the maximum degree $\bar{n}$ of the GEM coefficients are chosen based on combination of several factors such as the required accuracy, the spatial resolution of digital terrain and density models, and the numerical efficiently.

\section{References}

Anderson E. G., 1976: The effect of topography on solutions of Stokes' problem. Unisurv., S-14, Rep School of Surveying, University of New South Wales, Kensington, Australia.

Artemjev M. E., Kaban M. K., Kucherinenko V. A., Demjanov G. V., Taranov V. A., 1994: Subcrustal density inhomogeneities of the Northern Euroasia as derived from the gravity data and isostatic models of the lithosphere. Tectonophysics, 240, 248-280. 
Barnett C. T., 1976: Theoretical modeling of the magnetic and gravitational fields of an arbitrary shaped three dimensional body. Geophysics, 41, 1353-1364.

Bentsen M., Evensen G., Drange H., Jenkins A. D., 1999: Coordinate Transformation on a Sphere Using Conformal Mapping. Mon. Wea. Rev., 127, 2733-2740.

Bessel F. W., 1813: Auszug aus einem Schreiben des Herrn Prof. Bessel. Zach's Monatliche Correspondenz zur Beförderung der Erd- und Himmelskunde, XXVII, 80-85.

Bomford G., 1962: Geodesy ( $2^{\text {nd }}$ ed.). Oxford University Press, New York.

Bronstein I. N., Semendjajew K. A., 1977: Taschenbuch der Mathematik. Teubner B. G., Leipzig.

Chai Y., Hinze W. J., 1988: Gravity inversion of an interface above which the density contrast varies exponentially with depth. Geophysics, 53, 837-845.

Collette B. I., 1965: Chart for determining the gravity effect of two and three dimensional bodies bounded by arbitrary polygons. Geophys. Prospect., 13, 12-21.

Eshagh M., Sjöberg L. E., 2008: Impact of topographic and atmospheric masses over Iran on validation and inversion of GOCE gradiometric data. J. Earth Space. Phys., 34, $3,15-30$.

Eshagh M., Sjöberg L. E., 2009: Topographic and atmospheric effects on GOCE gradiometric data in a local north-oriented frame: A case study in Fennoscandia and Iran. Stud. Geophys. Geodaet., 53, 61-80.

Everest G., 1830: An account of the measurement of the arc of the meridian between the parallels of $18^{\circ} 3^{\prime}$ and $24^{\circ} 7^{\prime}$. The Royal Society, Printed by Cox J. L., London.

Gallardo-Delgado L. A., Perez-Flores M. A., Gomez-Trevino E., 2003: A versatile algorithm for joint inversion of gravity and magnetic data. Geophysics, 68, 949-959.

García-Abdeslem J., 1992: Gravitational attraction of a rectangular prism with depth dependent density. Geophysics, 57, 470-473.

García-Abdeslem J., 2005: Gravitational attraction of a rectangular prism with density varying with depth following a cubic polynomial. Geophysics, 70, J39-J42.

Götze H. J., Lahmeyer B., 1988: Application of three-dimensional interactive modelling in gravity and magnetics. Geophysics, 53, 8, 1096-1108.

Hagiwara Y., 1975: A new formula for evaluating the truncation error coefficient. J. Geod., 50, 2, 131-135, doi:10.1007/BF02522312.

Hamayun, Prutkin I., Tenzer R., 2009: The optimum expression for the gravitational potential of polyhedral bodies having a linearly varying density distribution. J. Geod., 83, 12, 1163-1170, doi:10.1007/s00190-009-0334-1.

Hansen R. O., 1999: An analytical expression for the gravity field of a polyhedral body with linearly varying density. Geophysics, 64, 1, 75-77.

Heck B., 2003: On Helmert's Methods of Condensation. J. Geod., 7, 155-170.

Heiskanen W. H., Moritz H., 1967: Physical geodesy. San Francisco, W. H. Freeman and Co.

Hinze W. J., 2003: Bouguer reduction density, why 2.67? Geophysics, 68, 5, 1559-1560.

Hobson E. W., 1931: The theory of spherical and ellipsoidal harmonics. Cambridge University Press, Cambridge. 
Holstein H., Ketteridge B., 1996: Gravimetric analysis of uniform polyhedra. Geophysics, 61, 2, 357-364.

Holstein H., Schürholz P., Starr A. J., Chakraborty M., 1999: Comparison of gravimetric formulas for uniform polyhedra. Geophysics, 64, 5, 1434-1446.

Holstein H., 2002a: Gravimagnetic similarity in anomaly formulas for uniform polyhedra. Geophysics, 67, 4, 1126-1133.

Holstein H., 2002b: Invariance in gravimagnetic anomaly formulas for uniform polyhedra. Geophysics, 67, 4, 1134-1137.

Holstein H., 2003: Gravimagnetic anomaly formulas for polyhedra of spatially linear media. Geophysics, 68, 1, 157-167.

Hurbbert M. K., 1948: A line integral method of computing the gravity effects of two dimensional masses. Geophysics, 13, 215-225.

Ivan M., 1990: Comment on "Optimum expression for computation of the gravity field of homogeneous polyhedral body" by V. Pohánka. Geophys. Prospect., 38, 331-332.

Karcol R., 2011: Gravitational attraction and potential of spherical shell with radially dependetn density. Stud. Geophys. Geodeat., 55, 21-34.

Kellogg O. D., 1929: Foundations of Potential Theory. Berlin, J. Springer.

Kühtreiber N., Kraiger G., Meurers B., 1989: Pilotstudie für eine neue Bouguer-Karte von Österreich. In: Österreichische Beiträge zu Meteorologie und Geophysik. Heft 2, Wien.

Kwok Y.-K., 1991: Gravity gradient tensor due to a polyhedron with polygonal facets. Geophys. Prospect., 39, 435-443.

Mader K., 1951: Das Newtonsche Raumpotential prismatischer Körper und seine Ableitungen bis zur dritten Ordnung. Sonderheft 11 der Österreichischen Zeitschrift für Vermessungswesen. Österreichischer Verein für Vermessungswesen, Wien.

Makhloof A. A., 2007: The use of topographic-isostatic mass information in geodetic application, Dissertation D98, Institute of Geodesy and Geoinformation, Bonn.

Martinec Z., Matyska C., Grafarend E. W., Vaníček P., 1993: On Helmert's $2^{\text {nd }}$ condensation method. Manusc. Geodaet., 18, 417-421.

Martinec Z., Vaníček P., 1994: Direct topographical effect of Helmert's condensation for a spherical geoid. Manusc. Geodaet., 19, 257-268.

Martinec Z., 1998: Boundary value problems for gravimetric determination of a precise geoid. Lecture notes in earth sciences, 73, Springer.

Mikuška J., Pašteka R., Marušiak I., 2006: Estimation of distant relief effect in gravimetry. Geophysics, 71, J59-J69.

Mollweide K. B., 1813: Aufösung einiger die Anziehing von Linien Flächen und Köpern betreffenden Aufgaben unter denen auch die in der Monatl Corresp Bd XXIV. S, 522. vorgelegte sich findet. Zach's Monatliche Correspondenz zur Beförderung der Erd- und Himmelskunde, Bd. XXVII, 26-38.

Molodensky M. S., Yeremeev V. F., Yurkina M. I., 1960: Methods for Study of the External Gravitational Field and Figure of the Earth. TRUDY Ts NIIGAiK, 131, Moscow, Geodezizdat; English translation: Israel Program for Scientific Translation, Jerusalem, 1962. 
Nagy D., Papp G., Benedek J., 2000: The gravitational potential and its derivatives for the prism. J. Geod., 74, 7/8, 552-560.

Novák P., 2000: Evaluation of gravity data for the Stokes-Helmert solution to the geodetic boundary-value problem. Technical Report, 207, Dept. of Geodesy and Geomatics Engineering, University of New Brunswick, Fredericton, Canada.

Novák P., 2010: High resolution constituents of the Earths gravitational field. Surv. Geoph., 31, 1, 1-21.

Novák P., Vaníček P., Martinec Z., Veronneau M., 2001: Effects of the spherical terrain on gravity and the geoid. J. Geod., 75, 9-10, 491-504.

Novák P., Grafarend E. W., 2005. The ellipsoidal representation of the topographical potential and its vertical gradient. J. Geod., 78, 11-12, 691-706.

Novák P., Grafarend E. W., 2006: The effect of topographical and atmospheric masses on spaceborne gravimetric and gradiometric data. Stud. Geophys. Geodaet., 50, 4, 549-582, doi:10.1007/s11200-006-0035-7.

Novák P., 2009: High resolution constituents of the Earth gravitational field. Surveys in Geophysics, 31, 1, 1-21.

Okabe M., 1979: Analytical expressions for gravity anomalies due to homogeneous polyhedral bodies and translation into magnetic anomalies. Geophysics, 44, 4, 730-741.

Paul M., 1973: A method of evaluating the truncation error coefficients for geoidal heights. Bulletin Geod., 110, 413-425.

Paul M. K., 1974: The gravity effect of a homogeneous polyhedron for three dimensional interpretation. Pure Appl. Geophys., 112, 553-561.

Petrovič S., 1996: Determination of the potential of homogeneous polyhedral bodies using line integrals. J. Geod., 71, 44-52.

Pick M., 1984: The gravitational effect of bodies with variable density. Stud. Geophys. Geodaet., 28, 381-392.

Pohánka V., 1988: Optimum expression for computation of the gravity field of a homogeneous polyhedral body. Geophys. Prospect., 36, 733-751.

Pohánka V., 1990: Replay to comment by M. Ivan. Geophys. Prospect., 38, 333-335.

Pohánka V., 1998: Optimum expression for computation of the gravity field of a polyhedral body with linearly increasing density. Geophys. Prospect., 46, 391-404.

Sjöberg L. E., 1998: The ellipsoidal corrections to the topographic geoid effects, J. Geod., 77, 804-808.

Sjöberg L. E., Nahavandchi H., 1999: On the indirect effect in the Stokes-Helmert method of geoid determination. J. Geod., 73, 87-93.

Sjöberg L. E., 2000: Topographic effects by the Stokes-Helmert method of geoid and quasi-geoid determinations. J. Geod., 74, 255-268.

Sjöberg L. E., 2001: Topographic and atmospheric corrections of gravimetric geoid determination with special emphasis on the effects of harmonics of degrees zero and one. J. Geod., 75, 283-290.

Sjöberg L. E., 2007: Topographic bias by analytical continuation in physical geodesy. J. Geod., 81, 345-350. 
Sünkel H., 1968: Global topographic-isostatic models. In: Mathematical and numerical techniques in Physical geodesy. Ed. Sünkel H., Lecture Notes in Earth Sciences, 7, Springer-Verlag, 417-462.

Takin M., Talwani M., 1966: Rapid computation of gravitational attraction of topography on a spherical Earth. Geophys. Prospect., 14, 119-142.

Talwani M., Worzel J. L., Landisman M., 1959: Rapid gravity computation for two dimension bodies with application to the Mendocino submarine fracture zone. J. Geophys. Res., 64, 49-59.

Talwani M., Ewing M., 1960: Rapid computation of gravitational attraction of three dimensional bodies of arbitrary shape. Geophysics, 25, 203-225.

Tenzer R., 2005: Spectral domain of Newton's integral. Bollettino di Geodesia e Scienze Affini, 2, 61-73.

Tenzer R., Moore P., Nesvadba O., 2007: Analytical solution of Newton's integral in Terms of Polar Spherical Coordinates. In.: Dynamic Planet Monitoring and Understanding a Dynamic Planet with Geodetic and Oceanographic Tools, (Eds.) Tregoning P., Rizos Ch., International Association of Geodesy Symposia, 2007, 130, 3, 410-415, doi:10.1007/978-3-540-49350-1_60.

Tenzer R., Novák P., Vajda P., Ellmann A., Abdalla A., 2011: Far-zone gravity field contributions corrected for the effect of topography by means of Molodensky's truncation coefficients. Stud. Geophys. Geodeat., 55, 55-71.

Tsoulis D., 1999: Spherical harmonic computations with topographic/isostatic coefficients. Reports in the series IAPG / FESG (ISSN 1437-8280), Report, 3, ISBN 3-934205-02-X, Institute of Astronomical and Physical Geodesy, Technical University of Munich.

Tsoulis D., 2001: A Comparison between the Airy-Heiskanen and the Pratt-Hayford isostatic models for the computation of potential harmonic coefficients. J. Geod., 74, 9, 637-643, doi:10.1007/s001900000124.

Tsoulis D., Petrovič S., 2001: On the singularities of the gravity field of a homogeneous polyhedral body. Geophysics, 66, 535-539.

Vaníček P., Najafi M., Martinec Z., Harrie L., Sjöberg L. E., 1995: Higher-degree reference field in the generalised Stokes-Helmert scheme for geoid computation. J. Geod., 70, 176-182.

Wild F., Heck B., 2004: Effects of topographic and isostatic masses in satellite gravity gradiometry. Proc. Second International GOCE User Workshop GOCE. The Geoid and Oceanography, ESA-ESRIN, Frascati, Italy, March 8-10, 2004 (ESA SP-569, June 2004), CD-ROM.

Werner R. A., Scheeres D. J., 1997: Exterior gravitation of polyhedron derived and compared with harmonic and mascon gravitation representations of asteroid 4769 Castalia. Celest. Mech. Dyn. Astron., 65, 313-344.

Zach F. X., 1811: Zach's Monatliche Correspondenz zur Beförderung der Erd- und Himmelskunde, Bd., XXVII, 522 p. 


\section{Appendix I: Analytical solution for cylinder}

The inner-zone integration element centered with respect to the observation point is represented by a cylinder mass volume of the radius $\tau=\mathrm{R} \psi_{\text {inner }}$ and the height $\eta=\Delta r$ for chosen small positive values of $\Delta r$ and $\psi_{\text {inner }}$ (see Fig. I), where $\psi_{\text {inner }}$ is the maximum spherical distance of the inner zone (see Eq. 1). The axis of the cylinder coincides with the r-axis of the observation point. The actual integral element around the computation point is very close in shape to this cylindrical approximation for the regions near the surface of the Earth because in this case $r>>\Delta r$ and $\psi_{\text {inner }}<<1$. We need to distinguish three separate cases depending on the location of the observation point within the cylinder. If the observation point is on top/bottom of cylinder (i.e., points $\mathrm{A}$ and $\mathrm{C}$ in Fig. I), the potential reads (e.g., Heiskanen and Moritz, 1967).

$V(A)=\pi \mathrm{G} \rho\left[\eta \sqrt{\eta^{2}+\tau^{2}}+\tau^{2} \log \left(\frac{\sqrt{\eta^{2}+\tau^{2}}+\eta}{\tau}\right)-\eta^{2}\right]$.

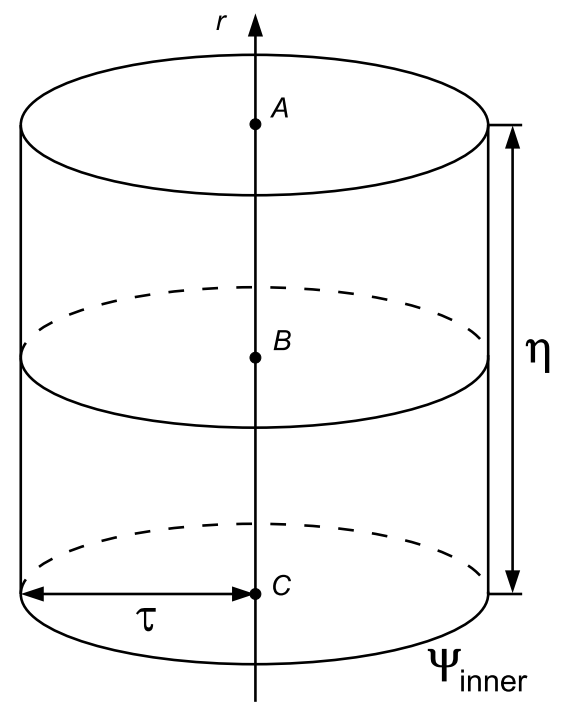

Fig. I. The cylinder mass volume centered with respect to the r-axis of the observation point. The location of the observation point is: $\mathrm{A}$ - on top of cylinder, $\mathrm{B}$ - in middle of cylinder, and $\mathrm{C}-$ at bottom of cylinder. 
If the observation point is identical with a geometric centre of cylinder (i.e., point B in Fig. I), we obtain

$V(B)=\pi \mathrm{G} \rho\left[\frac{\eta}{2} \sqrt{\eta^{2}+4 \tau^{2}}+\tau^{2} \log \left(\frac{\sqrt{\eta^{2}+4 \tau^{2}}+\eta}{\sqrt{\eta^{2}+4 \tau^{2}}-\eta}\right)-\frac{\eta^{2}}{2}\right]$.

Similarly, we define the gravitational acceleration $g$ of the cylinder mass volume for three cases. If the observation point is on top of cylinder, we have (e.g., Heiskanen and Moritz, 1967)

$g(A)=2 \pi \mathrm{G} \rho\left(\sqrt{\tau^{2}+\eta^{2}}-\tau-\eta\right)$.

If the observation point is in middle of cylinder, the acceleration becomes zero. Hence

$g(B)=0$.

If the observation point is at bottom of cylinder, we have (ibid.)

$g(C)=2 \pi \mathrm{G} \rho\left(\tau+\eta-\sqrt{\tau^{2}+\eta^{2}}\right)$. 